Article

\title{
Silk: Optical Properties over 12.6 Octaves THz-IR-Visible-UV Range
}

\author{
Armandas Balčytis ${ }^{1,2, *, \dagger}$, Meguya Ryu ${ }^{3,+}$, Xuewen Wang ${ }^{1}$, Fabio Novelli ${ }^{1, \ddagger}$, \\ Gediminas Seniutinas ${ }^{1, \S}$, Shan Du ${ }^{4}$, Xungai Wang ${ }^{4}$, Jingliang Li ${ }^{4}$, Jeffrey Davis ${ }^{1}$, \\ Dominique Appadoo ${ }^{5}$, Junko Morikawa ${ }^{3, *}$ and Saulius Juodkazis ${ }^{1,6}$
}

1 School of Science, Faculty of Engineering and Industrial Sciences, Swinburne University of Technology, Hawthorn, VIC 3122, Australia; xuewenwang@swin.edu.au (X.W.); fabio.novelli@ruhr-uni-bochum.de (F.N.); gdseniutinas@gmail.com (G.S.); jdavis@swin.edu.au (J.D.); saulius.juodkazis@gmail.com (S.J.)

2 Department of Laser Technologies, Center for Physical Sciences and Technology, Savanoriu Ave. 231, LT-02300 Vilnius, Lithuania

3 Tokyo Institute of Technology, Meguro-ku, Tokyo 152-8550, Japan; ryu.m.ab@m.titech.ac.jp

4 Australian Future Fibres Research and Innovation Centre, Institute for Frontier Materials, Deakin University, Geelong, VIC 3220, Australia; shand@deakin.edu.au (S.D.); xungai.wang@deakin.edu.au (X.W.); jingliang.li@deakin.edu.au (J.L.)

5 Australian Synchrotron, Blackburn Road, Clayton, VIC 3168, Australia; dom.appadoo@synchrotron.org.au

6 Melbourne Centre for Nanofabrication, the Victorian Node of the Australian National Fabrication Facility, 151 Wellington Rd., Clayton, VIC 3168, Australia

* Correspondence: abalcytis@swin.edu.au (A.B.); morikawa.j.aa@m.titech.ac.jp (J.M.)

+ These authors contributed equally to this work.

$\ddagger$ Current address: Ruhr-University Bochum, 44801 Bochum, Germany.

$\S$ Current address: Paul Scherrer Institut, CH-5232 Villigen PSI, Switzerland.

Academic Editor: Armando J. D. Silvestre

Received: 30 January 2017; Accepted: 23 March 2017; Published: 28 March 2017

\begin{abstract}
Domestic (Bombyx mori) and wild (Antheraea pernyi) silk fibers were characterised over a wide spectral range from THz $8 \mathrm{~cm}^{-1}(\lambda=1.25 \mathrm{~mm}, f=0.24 \mathrm{THz})$ to deep-UV $50 \times 10^{3} \mathrm{~cm}^{-1}$ $(\lambda=200 \mathrm{~nm}, f=1500 \mathrm{THz}$ ) wavelengths or over a 12.6 octave frequency range. Spectral features at $\beta$-sheet, $\alpha$-coil and amorphous fibroin were analysed at different spectral ranges. Single fiber cross sections at mid-IR were used to determine spatial distribution of different silk constituents and revealed an $\alpha$-coil rich core and more broadly spread $\beta$-sheets in natural silk fibers obtained from wild Antheraea pernyi moths. Low energy T-ray bands at 243 and $229 \mathrm{~cm}^{-1}$ were observed in crystalline fibers of domestic and wild silk fibers, respectively, and showed no spectral shift down to $78 \mathrm{~K}$ temperature. A distinct $20 \pm 4 \mathrm{~cm}^{-1}$ band was observed in the crystalline Antheraea pernyi silk fibers. Systematic analysis and assignment of the observed spectral bands is presented. Water solubility and biodegradability of silk, required for bio-medical and sensor applications, are directly inferred from specific spectral bands.
\end{abstract}

Keywords: silk; fibroin; biopolymer; terahertz; spectroscopy; solubility; proteins; biodegradable polymers

\section{Introduction}

Spectral properties at sub- $1 \mathrm{~mm}$ wavelengths at around and below terahertz frequencies ( $1 \mathrm{THz}=10^{12} \mathrm{~Hz}$, corresponding to $\approx 33 \mathrm{~cm}^{-1}$ in wavenumbers) are important for understanding materials with bio-medical relevance [1]. For example, terahertz absorbance is related to conformation and structure of saturated fatty acids with long alkane chains, whose vibration frequency depends on 
the length $l$ of the chain as $v=\sqrt{\frac{E}{\rho}} /(2 l c)\left[\mathrm{cm}^{-1}\right]$ for density $\rho$, Young modulus $E$, and speed of light $c$-as demonstrated for polymethylene [2]. Those longitudinal accordion modes (LAMs) populate a $1-100 \mathrm{~cm}^{-1}$ low energy vibration window, edging towards low wavenumbers for long chains. Of relevance to material science are first order solid-solid phase transitions in alkane crystals-the rotator phases [3]—occurring just a few degrees below melting point when the crystalline order still exists; however, low energy rotations of molecules become allowed [4]. In glasses, low frequency Raman spectra exhibit the $10-50 \mathrm{~cm}^{-1}$ Boson peak, which is another example of low energy phenomena due to rearrangement of density of states in amorphous materials [5].

In protein based materials, a variety of molecular ordering and interactions occur, which, in turn, define their properties. It was shown that formation of protein fibrils can be monitored at the $\mathrm{THz}$ spectral window [6]. Silk fibers offer a good example of the complexity of protein materials as they have amorphous and crystalline structural components with proteins forming a 3D network of random $\alpha$-coils and metastable $\beta$-turns (Silk I) together with a crystalline $\beta$-sheet phase (Silk II) [7]. Such composition results in a set of important properties such as a high mechanical strength, optical transparency and waveguiding [8] as well as biocompatibility and biodegradability $[9,10]$.

Mechanisms controlling crystallisation of protein coils via ordered hydrogen bonding and their unzip-decomposition at different annealing temperatures, laser and electron beam exposure conditions are of interest for applications in material science and bio-medical fields [11-13]. Silk can be used as a bio-compatible scaffold [14] with water solubility dependent on its crystallinity [15]. Furthermore, the biodegradability and elasticity of silk make it an attractive platform for the creation of next-generation biocompatible and flexible optoelectronic devices [16]. Any active functional materials used in such applications alongside silk would have to exhibit conformable mechanical characteristics. Two-dimensional transition metal oxides, with their wide variety of controllable physical properties, are especially promising in this regard [17]. While the field is still nascent, silk fibroin was already demonstrated to be a viable template in preparing metal oxide composite nanomaterials for lithium-ion battery anodes $[18,19]$. However, in order to tune the morphology of nanomaterials created in this way, control over the self-assembly behavior of hierarchical fibroin structure is required [20].

Photo-thermal control of $\beta$-sheet formation in silk could provide a way to make silk-resists as well as 3D bio-scaffolds, and would help to understand protein crystallisation mechanisms relevant to the $\beta$-sheet plaque formation in Alzheimer's disease. It was demonstrated using on-chip calorimetry that, through a fast $2000 \mathrm{~K} / \mathrm{s}$ thermal quenching of molten silk, an amorphous phase (water soluble) can be recovered [21]. However, fast thermal quenching is hampered by the rather low thermal diffusivity of silk $\alpha_{T} \simeq 1.5 \times 10^{-7} \mathrm{~m}^{2} / \mathrm{s}$ [22], likewise attributable to its complex structure. Similarly, the interaction of light with the multiple constituent structural components of silk fibers at frequencies spanning from far-IR to UV spectral ranges has to be well understood for a wide range of applications.

Here, transmittance measurements of silk over a broad spectral range from $\mathrm{THz}$ wavelengths (T-ray) until deep UV are reported and spectral signatures of the constituent components of silk: $\beta$-sheets, $\alpha$-coils and amorphous fibroin are analysed. Since the spectral signatures characteristic of the silk building blocks are present at very different wavelengths, a comprehensive analysis over the broad spectral range had to be made. Water solubility of silk can be inferred from the spectral properties and is essential for future applications of silk in wearable electronics, implants, and sensors.

\section{Materials and Methods}

\subsection{Spectroscopy Setups and Techniques}

The $\mathrm{THz} /$ Far-IR Beamline at the Australian synchrotron was used to characterise silk in the $40-600 \mathrm{~cm}^{-1}$ spectral range. The beamline is equipped with a Bruker IFS 125/HR Fourier Transform (FT) spectrometer (Bremen, Germany) and OPUS 6.5 software (Bruker Optik GmbH, Ettlingen, Germany) was used for initial data analysis. Up to 100 spectral scans were captured and averaged to 
improve signal-to-noise $(\mathrm{S} / \mathrm{N})$ ratio. A liquid nitrogen cryostat was used to measure silk transmittance, $T$, down to $\sim 77 \mathrm{~K}$ temperature.

For the largest T-ray wavelengths in the $8-80 \mathrm{~cm}^{-1}$ spectral window, femtosecond time domain spectroscopy (TDS) was used due to the higher $\mathrm{S} / \mathrm{N}$ ratio as compared to synchrotron T-ray radiation. Almost a single-cycle and 1 ps long THz-fields are generated in a $0.6 \% \mathrm{MgO}$ doped $\mathrm{LiNbO}_{3} \mathrm{crystal}$ by optical rectification of amplified laser pulses $[23,24]$. The radiation is focused onto the sample by an off-axis parabolic mirror with a focal length of $100 \mathrm{~mm}$ and imaged with two additional identical mirrors onto the detection crystal. The single-cycle fields are detected via electro-optical sampling in a $500 \mu \mathrm{m}$ thick ZnTe [25].

The amplitude and phase-resolved fields transmitted through the silk samples, as well as through a reference (air), are Fourier transformed to give frequency-dependent amplitudes as well as their phases. If the sample thickness is well defined and only the first Fresnel coefficient can be considered, the phase difference of these Fourier transforms is directly related to the real part of the index of refraction, whereas the ratio of the field magnitudes yields the absorption coefficient. In the case of silk fibers, however, the sample thickness is not well defined, so only the absorbance, $A$, can be unambiguously deduced, calculated as $-\lg T$, where $T$ is the field transmittance corresponding to the ratio of the Fourier magnitudes. The fields transmitted by a reference and by the sample are alternatively acquired 50 times to give the standard deviation shown as error bars in Figure 1.

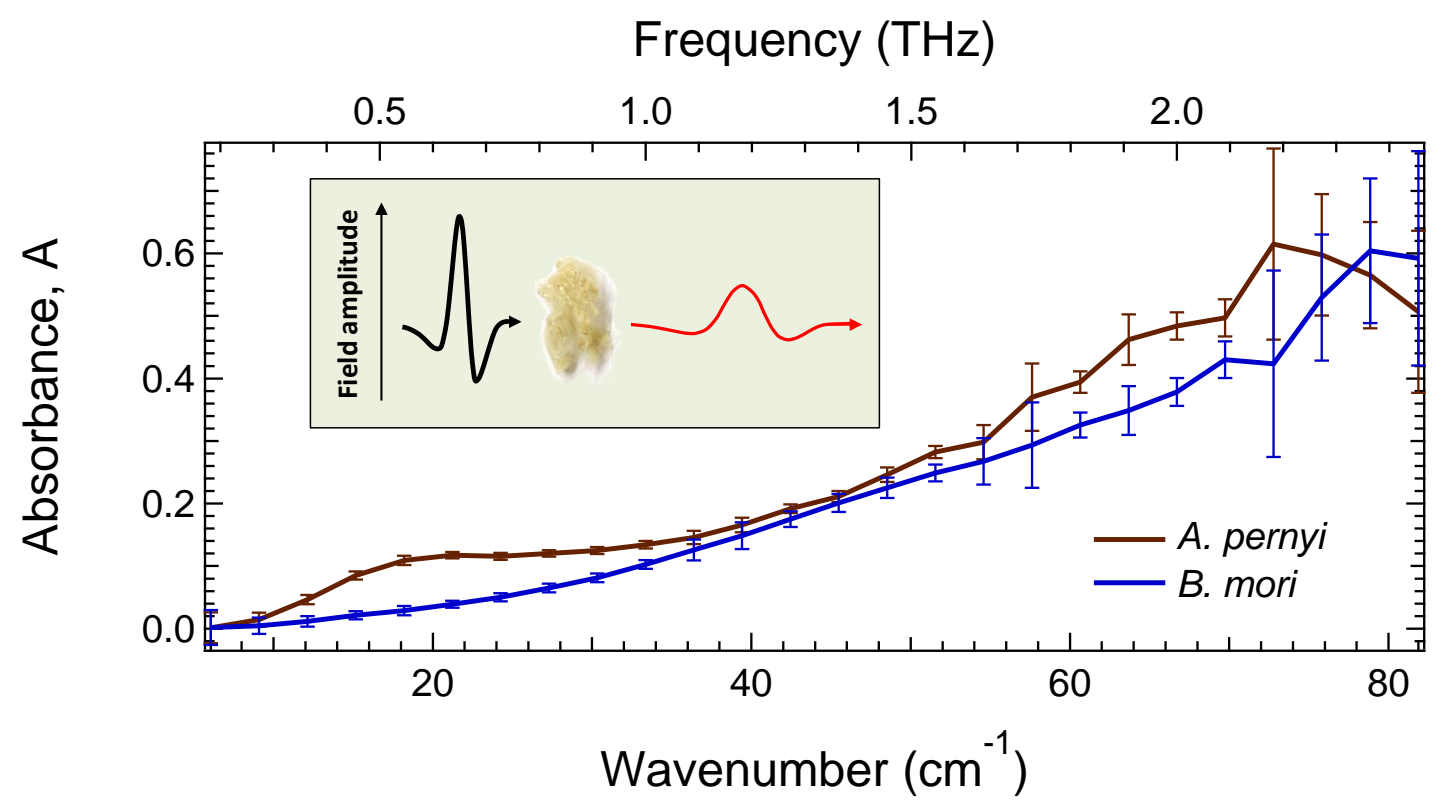

Figure 1. Absorbance, $A=-\lg T$, spectra of $B$. mori and $A$. pernyi silk fibers measured by time domain spectroscopy (TDS). The transmittance, $T$, is calculated as the ratio of the magnitudes of the Fourier transforms of the field transmitted through the sample and through a reference (air). Error bars represent one standard deviation from 50 measurements.

Shorter wavelength characterisation was carried out with a UV-Vis spectrometer (Lambda 1050 UV/Vis, PerkinElmer, Waltham, MA, USA) by measuring total transmittance and reflectance of fibers in a $150 \mathrm{~mm}$ integrating sphere geometry. Photoluminescence excitation spectra were collected using a fluorescence spectrometer LS55 (PerkinElmer), whereas a FT-IR spectrometer (Vertex70, Bruker) was used for far-IR-to-near-IR transmittance measurements. Spectral ranges of the selected tools allowed for continuously covering an unprecedentedly wide spectral range from T-rays to deep-UV.

The measured spectral properties over a large range of wavelengths have different contributions of Rayleigh scattering, which is proportional to $\lambda^{-4}$ and Mie scattering, which becomes significant for size 
parameter $x=(2 \pi / \lambda) d>1$, given here for spherical particles of radius $d$ for which analytical solutions are known. Mie scattering has very strong polarization and angular dependence [26], which makes absorbance measurements sensitive to the numerical aperture. Furthermore, Mie scattering has stronger intensity fluctuations for more absorbing materials. For direct estimation of Mie scattering of realistic samples, a large number of silk fibers would be required; however, this was out of the scope of the current study.

\subsection{Silk Samples}

Silk samples were prepared from domestic Bombyx mori and wild Antheraea pernyi species of silk worms. A. pernyi silkworm cocoons were obtained from Liaoning province, China. B. mori was collected from the silk rearing house in Jiangsu province, China. The silk fiber from B. mori has superior elasticity and toughness due to the way the disordered fibroin matrix is reinforced by glycine and alanine based $\beta$-sheets [27]. However, $A$. pernyi has a different primary structure of fibroin, composed of alternating appearances of large repetitive poly-alanine blocks and glycine rich regions [28]. This is in contrast to B. mori but has distinct similarities to spider dragline silk [29]. Furthermore, A. pernyi survives at the lowest temperatures among all silk moths. Raw cocoons were degummed three times using $0.5 \% \mathrm{Na}_{2} \mathrm{CO}_{3}$ solution at $98^{\circ} \mathrm{C}$ for $1 \mathrm{~h}$ for $A$. pernyi cocoon, but $30 \mathrm{~min}$ for B. mori cocoon $[30,31]$. Finally, the degummed silk fibers were thoroughly rinsed with warm deionised water $\left(60^{\circ} \mathrm{C}\right)$ prior to being dried in air.

Fibroin extraction from degummed $B$. mori silk fibers was done by first dissolving them in a 1:8:2 molar ratio ternary mixture of $\mathrm{CaCl}_{2} / \mathrm{H}_{2} \mathrm{O} / \mathrm{CH}_{3} \mathrm{CH}_{2} \mathrm{OH} 65^{\circ} \mathrm{C}$. Then, the solution was dialysed against ultra-pure water with dialysis tubing cellulose membrane (molecular weight cut-off $14 \mathrm{kDa}$, Sigma-Aldrich Co., St. Louis, MO, USA) at room temperature for 4 days. Finally, silk fibroin was regenerated by lyophilizing the dialysed solution. The same procedure was used in prior work to make a fibroin-based electron beam resist [22].

Silk fibroin samples spectroscopically investigated in this work allow for comparisons between $\beta$-sheet rich, high-crystallinity, hence water-insoluble silk fibers, and soluble amorphous fibroin without significant $\beta$-sheet content. Furthermore, fibers originating from different silkworm species exhibiting variations in secondary structure are also probed in a broad spectral range.

\section{Results and Discussion}

Different spectral ranges of silk absorbance/transmittance have been probed using different methods; however, there was always an overlap between two adjacent spectral ranges. Absorbance at the longest T-ray wavelengths at $8-80 \mathrm{~cm}^{-1}(0.24-2.4 \mathrm{THz} ; 1.25 \mathrm{~mm}-125 \mu \mathrm{m})$ was measured with TDS while, for the shorter wavelengths up to $600 \mathrm{~cm}^{-1}(18 \mathrm{THz} ; 16.6 \mu \mathrm{m})$, synchrotron beamline was used. At wavenumbers above $600 \mathrm{~cm}^{-1}$, investigation was conducted by means of FT-IR spectroscopy up to $4000 \mathrm{~cm}^{-1}(120 \mathrm{THz} ; 2.5 \mu \mathrm{m})$, at which point near-IR-to-visible spectroscopy took over up to wavenumbers of $33,333 \mathrm{~cm}^{-1}$ (roughly corresponding to $1000 \mathrm{THz}$ in frequency and $300 \mathrm{~nm}$ in wavelength). Finally, photoluminescence excitation spectroscopy aided in providing information at deeper UV wavelengths up to $50,000 \mathrm{~cm}^{-1}(1500 \mathrm{THz} ; 200 \mathrm{~nm})$.

\subsection{T-Rays}

The terahertz frequency range is associated with low frequency macromolecular motions strongly related to the dynamics and conformational changes of proteins and peptides [32]. Hence, it is useful in probing the secondary and to some extent the primary structure of complex biomaterials. Figure 1 shows absorbance spectra measured with the TDS technique. Distinct $20 \mathrm{~cm}^{-1}$ and $67 \mathrm{~cm}^{-1}(0.6 \mathrm{THz}$ and $2 \mathrm{THz}$ ) bands were observed in $A$. pernyi silk fibers.

Figure 2 shows room temperature (RT) far-IR synchrotron radiation absorbance, $A$, spectra of different silk samples. Sericin-free crystalline fibers of B. mori and A. pernyi silk have slightly different spectral positions of the absorption bands. Amorphous water soluble fibroin had a spectrally broader 
absorption at $\sim 115 \mathrm{~cm}^{-1}$. All the spectra were measured with fibers or film (amorphous) suspended over a hole though which a 3-mm-diameter T-ray beam was propagating. There were no artifacts due to normalization to the background T-ray transmittance at those spectral locations.

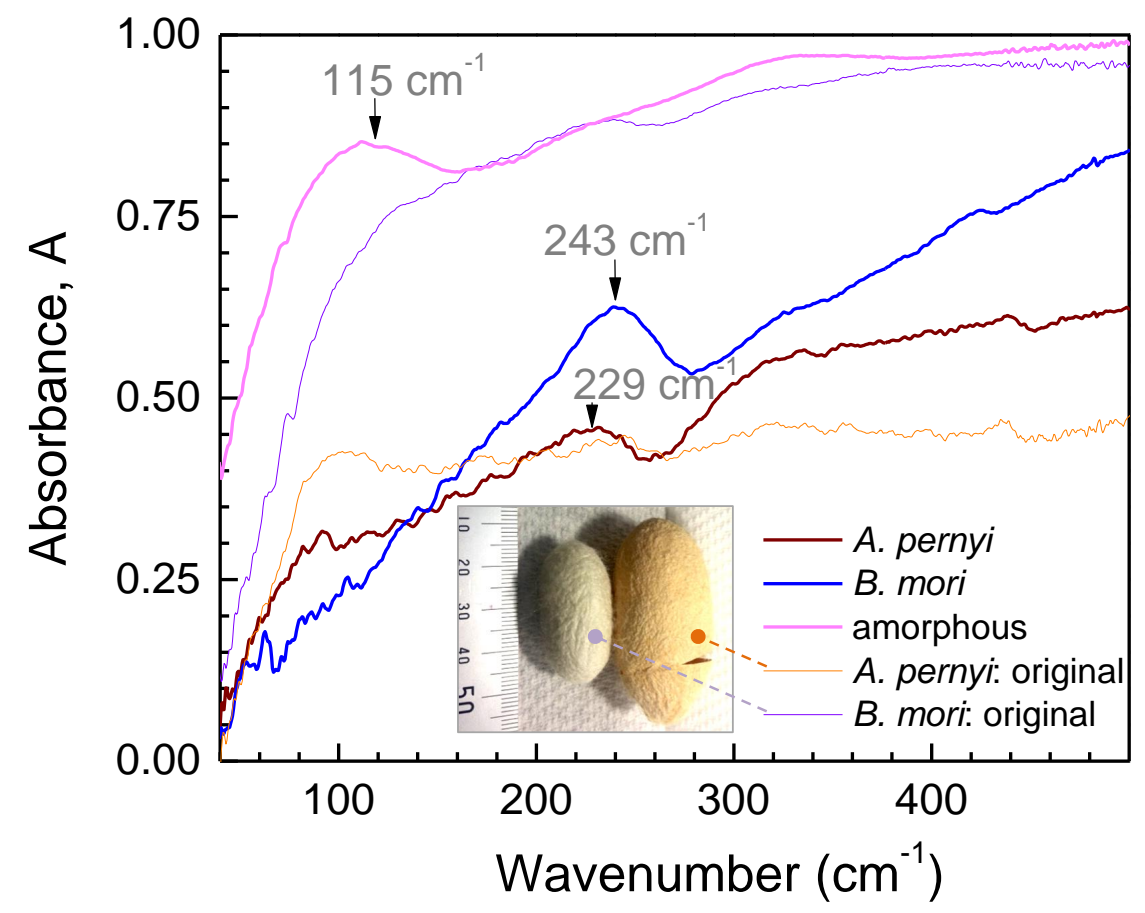

Figure 2. T-ray absorbance, $A$, spectra of domestic (degummed B. mori) and wild (degummed A. pernyi) silk fibers together with amorphous fibroin extracted from B. mori silk and fibers from non degummed, as harvested, silk cocoons with sercin. All spectra were measured at room temperature (RT). Data averaged over 100 scans.

Absorbance spectra of degummed B. mori and A. pernyi silk fibers as well as amorphous fibroin from B. mori subjected to hot plate annealing at $250{ }^{\circ} \mathrm{C}$, i.e., at the onset of thermal degradation [33], for varying durations are shown in Figure 3. Degradation of silk with observable darker coloration was consistent with high temperature oxidation. However, for the crystalline $\beta$-sheet rich fibers, there were no strong changes in the absorption bands at $\sim 243 \pm 15 \mathrm{~cm}^{-1}$ (B. mori) and $\sim 229 \pm 15 \mathrm{~cm}^{-1}$ (A. pernyi) silk (Figure 3a,b). This is consistent with $\beta$-sheets exhibiting a higher resilience to thermal decomposition than random coil structures. Furthermore, at elevated temperatures, metastable fibroin fractions in the secondary structure of silk are liable to transform into the stable $\beta$-sheet crystals [33]. Hence, the observed variations in T-ray absorbance spectra for the different durations of heating at the lower edge of the thermal degradation range can tentatively be related to a decrease of water content or preferential decomposition of the amorphous regions in comparison with $\beta$-sheets. Further support is provided by spectral variations due to thermal degradation in amorphous B. mori fibroin. It experienced a strong reduction of absorbance throughout the investigated range, especially at larger wavenumbers and a disappearance of the $\sim 125 \pm 15 \mathrm{~cm}^{-1}$ band (Figure 3c). 

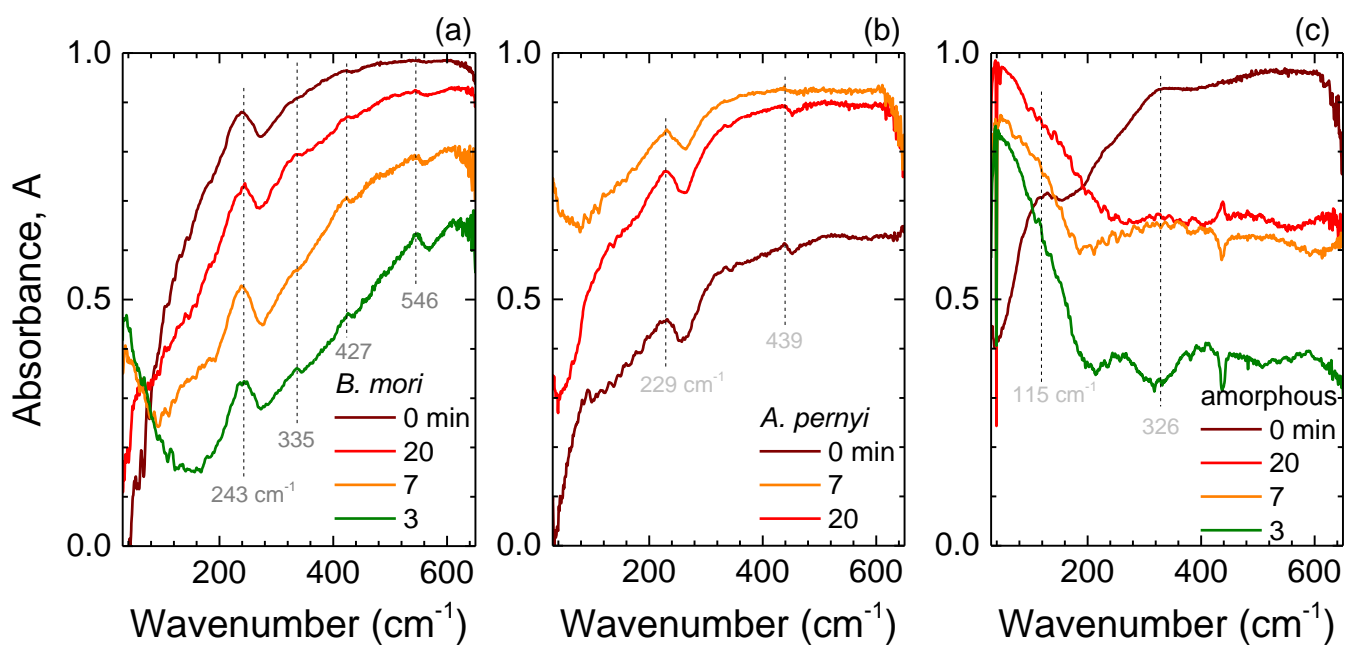

Figure 3. Absorbance, $A$, spectra of (a) B. mori silk fibers; (b) A. pernyi silk fibers and (c) amorphous silk fibroin samples annealed on a hot plate for 3, 7, $20 \mathrm{~min}$ at cleanroom class 1000 conditions. Amount of silk in each sample was the same $(0.6 \mathrm{mg})$ during annealing; however, for IR spectral measurements, a small amount of fibers was placed over the 3-mm-diameter aperture. Data averaged over 100 scans. Temperature of the hot plate was $250^{\circ} \mathrm{C}$.

The spectral range in the vicinity of $240 \mathrm{~cm}^{-1}$ is also associated with water absorption bands. In particular, at room temperature, liquid water has a broad spectral feature at $200 \mathrm{~cm}^{-1}$, assigned to the stretching of intermolecular hydrogen bonds, and another at $700 \mathrm{~cm}^{-1}$, related to librational motions [34,35]. Therefore, it is important to ascertain whether the observed peaks are related to water content. As silk is heated, water removal by evaporation efficiently proceeds in the range from $70{ }^{\circ} \mathrm{C}$ to $\sim 200{ }^{\circ} \mathrm{C}$ [36]. Therefore, if the peaks at around $240 \mathrm{~cm}^{-1}$ were related to water, they would be expected to gradually disappear in the thermally treated samples, which was not observed in experiment (Figure 3). Conversely, water can also be revealed by ice formation and a corresponding emergence of a spectrally narrow feature at around $230 \mathrm{~cm}^{-1}$ when cooled towards liquid nitrogen (77 K) temperature $[37,38]$. The crystalline and amorphous samples were cooled and their absorbance spectra measured (Figure 4). A spectrally narrow ice band was not observed. There were no spectral shifts with lowering temperature and only slight narrowing of the characteristic absorbance bands, best recognizable in A. pernyi silk (Figure $4 b$ ). Thereby, water can be ruled out as the cause of the observed T-ray absorbance peaks.

The far-IR spectra of natural silk fibers are made more complex by the heterogeneous arrangement of their constituent proteins. However, for B. mori silk, in addition to the dominant $243 \mathrm{~cm}^{-1}$ absorption band, three other peaks at 335,427 , and $546 \mathrm{~cm}^{-1}$ can be discerned, especially for heat-treated samples with reduced water content. Four bands have been previously observed in a similar spectral vicinity at $250,328,427$, and $553 \mathrm{~cm}^{-1}$, respectively, for $\beta$-sheet rich fibroin films [39]. In contrast, A. pernyi silk fibers have far-IR absorbance spectra with two discernible peaks-at 229 and $439 \mathrm{~cm}^{-1}$. The prevalence of poly-alanine in wild silks provides a hint to their possible assignments. Stretched $\beta$-form of poly-L-alanine exhibits absorbance peaks at 240 and $432 \mathrm{~cm}^{-1}$ [39]. Lastly, amorphous B. mori fibroin spectra have a broad peak at $\sim 115 \mathrm{~cm}^{-1}$ as well as a shoulder at $326 \mathrm{~cm}^{-1}$, consistent with the $\alpha$-helix state of poly(alanine-glycine) (Ala-Gly) [39]. In addition, the observed variations of these spectral features due to heat treatment would lend further credence to their assignment to the metastable Silk I state.

Overall, the peaks observed in the fibers at far-infrared exhibit slight shifts with respect to their extracted counterparts; furthermore, they are generally broad and not well defined. This lends credence to the view that, at the terahertz range, ensembles of resonances characterising the secondary structure 
of proteins are detected [40]. This, in turn, opens new possibilities to probe structural properties at T-ray bands beyond the scope of typical spectroscopic approaches.

(a)

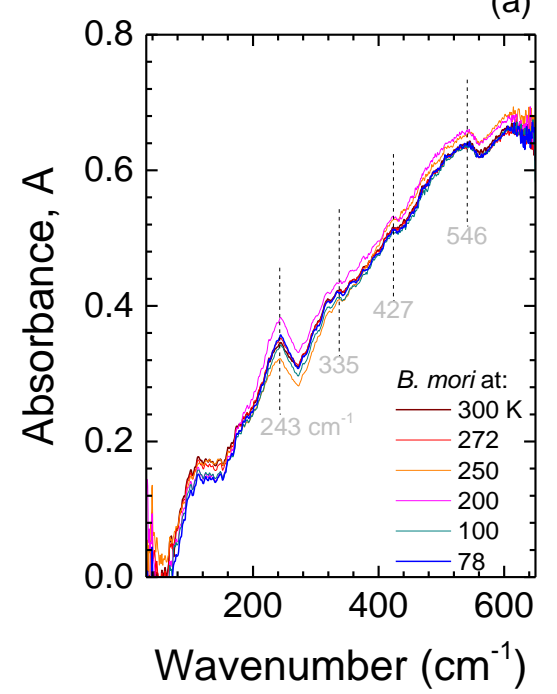

(b)

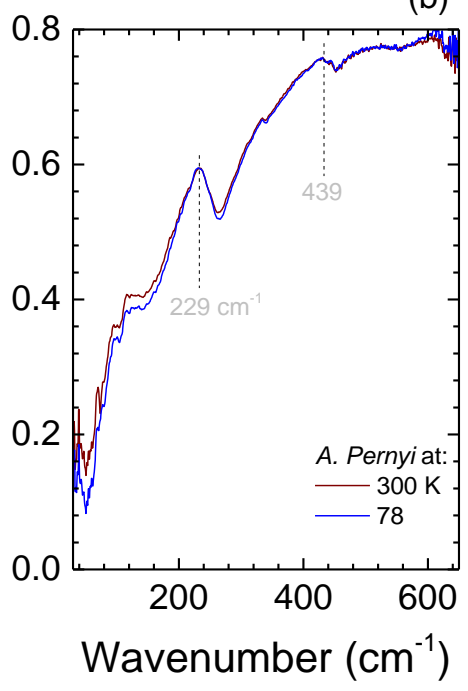

(c)

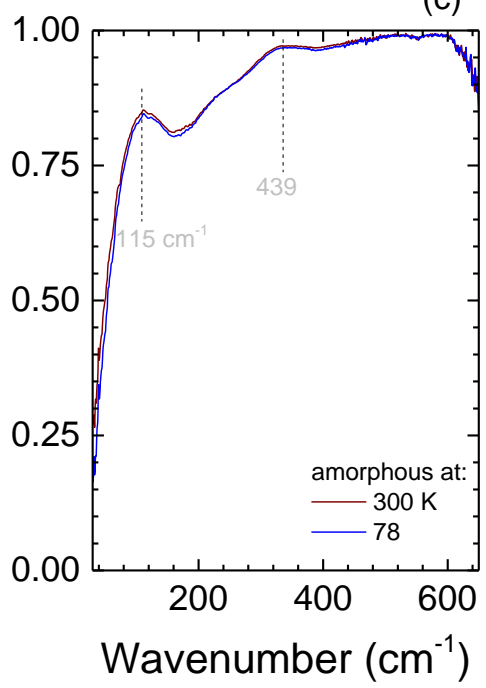

Figure 4. Temperature dependence of absorbance, $A$, spectra of (a) B. mori degummed silk fibers; (b) A. pernyi degummed silk fibers and amorphous (c) B. mori fibroin samples at different temperatures using a liquid nitrogen cryostat. Data averaged over 100 scans. Note different $A$ scales between (a-c).

\subsection{Mid-IR and IR Range}

The spectral range in excess of $600 \mathrm{~cm}^{-1}$ wavenumbers, beyond synchrotron T-ray scope, was probed using mid-IR FT-IR spectroscopy and imaging. Fibroin based fibers have been extensively studied in this wavelength region [41,42]; therefore, measurements provide a way for verification against the established body of work. Figure 5 shows cross polarised images of the samples. Degummed A. pernyi silk fibers were $\sim 50 \mu \mathrm{m}$ in diameter and approximately $2-3$ times thicker than B. mori silk fibers.

Figure 6 shows mid-IR spectra of domestic B. mori and wild A. pernyi species' silk fibers as well as of extracted fibroin, taken using the attenuated total reflection (ATR) technique. The $\mathrm{N}-\mathrm{H}$ stretching, alkyl, and Amide bands represent the most prominent spectral features for all three samples. Some absorbance peaks of significant interest are the $961 \mathrm{~cm}^{-1}$ band attributed to poly-alanine (Ala) ${ }_{n}$ in $\beta$-sheets, observed primarily in wild silks [41]. Conversely, domesticated B. mori derived white silk fibers exhibit different, slightly subdued features at 975 and $998 \mathrm{~cm}^{-1}$, consistent with their (Ala-Gly) ${ }_{n}$ based $\beta$-sheets $[41,43]$. The component at $1014 \mathrm{~cm}^{-1}$ is associated with random coil (Ala-Gly) sequences that have many interruptions by tyrosine (Tyr), valine (Val) or other peptide chains [43], and, as expected, it is most pronounced in the spectrum of disordered amorphous fibroin. Peaks in the vicinity of $1052 \mathrm{~cm}^{-1}$ fall within the $v(\mathrm{C}-\mathrm{C})$ skeletal range and are expected to have variations in different silks due to the presence of small amounts of other amino-acids in addition to Ala and Gly - with the $1052 \mathrm{~cm}^{-1}$ peak being more closely associated with $\alpha$-coils and metastable $\beta$-turns (i.e., Silk I), whereas $1052 \mathrm{~cm}^{-1}$ was shown to be related to anti-parallel $\beta$-sheets (Silk II) [43]. The band at $1165 \mathrm{~cm}^{-1}$ is common among all silks and assigned to $v\left(\mathrm{~N}-\mathrm{C}_{\alpha}\right)$ vibrations [41]. Its width is generally larger for domesticated silk species and even more so for amorphous fibroin, indicating more variety in conformation states. 
(a)

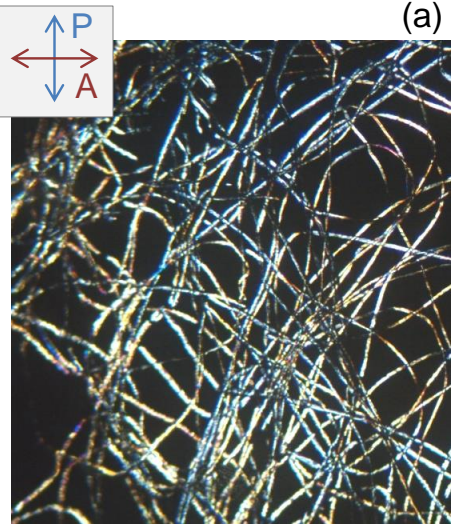

B. mori

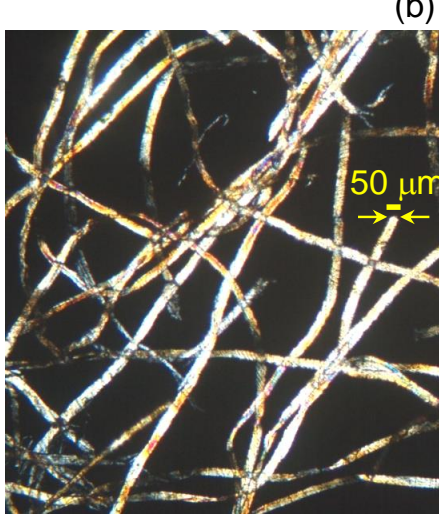

A. pernyi (b)

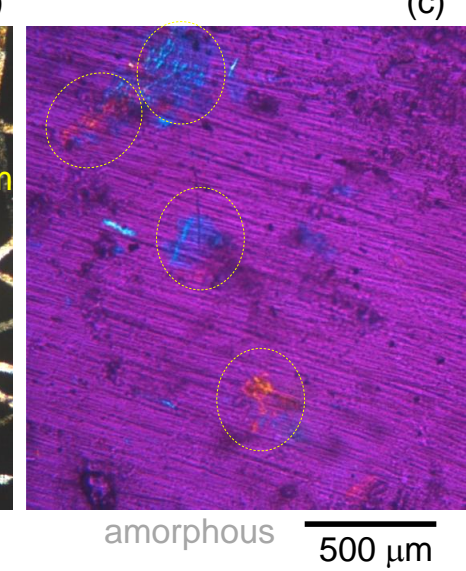

Figure 5. Optical cross-polarised optical images of degummed fibers of B. mori (a); A. pernyi (b); and amorphous fibroin from B. mori silk (c). Samples were compressed with KBr powder to form $\sim 100 \mu \mathrm{m}$ thick pallets. Marked regions in (c) show locations of amorphous fibroin. An additional $\lambda / 2$-plate was introduced to set the best background contrast with the same color corresponding to the same difference in the optical path length.

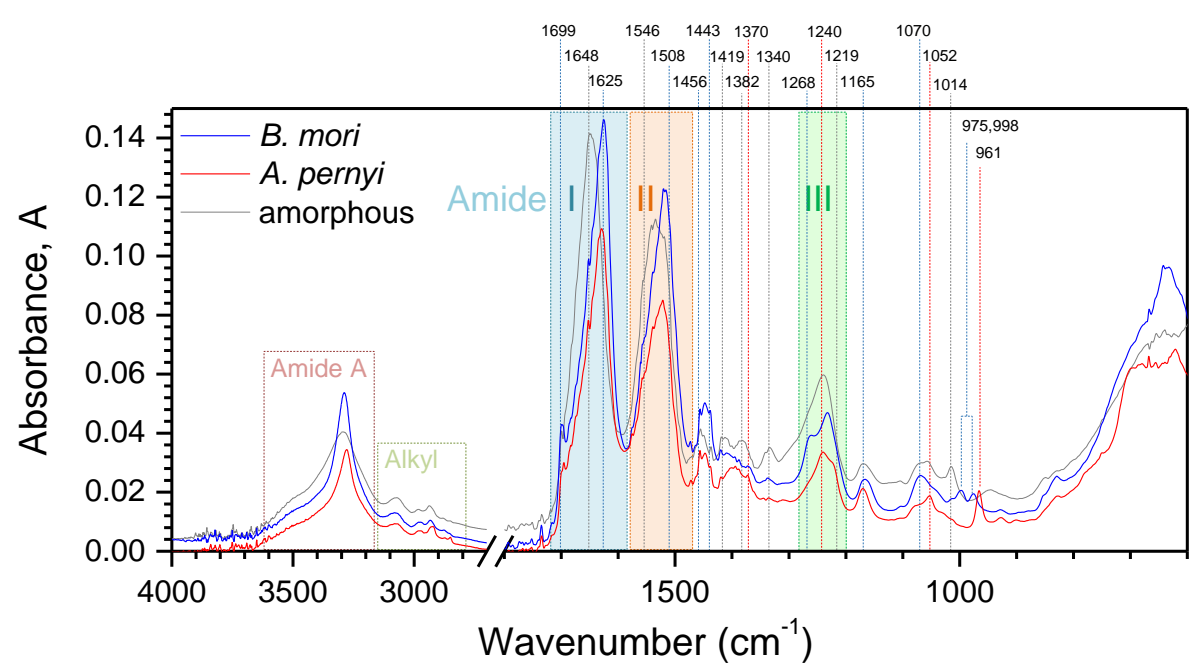

Figure 6. Absorbance, $A$ spectra of B. mori and A. pernyi silk fibers and amorphous fibroin powder measured using a diamond window and attenuated total reflection (ATR) method (Alpha, Bruker).

The Amide III band ranging from $1200 \mathrm{~cm}^{-1}$ to $1300 \mathrm{~cm}^{-1}$ is a complex spectral region with overlapping contributions from various amide side-chains in differing conformations [44]. The components are typically assigned for $\beta$-sheets at $1219 \mathrm{~cm}^{-1}$, random coil at $1240 \mathrm{~cm}^{-1}$ and $\alpha$-coils at $1268 \mathrm{~cm}^{-1}$ [41], with B. mori exhibiting a peak at $1232 \mathrm{~cm}^{-1}$ consistent with the Silk I form. Finally, a feature at $1308 \mathrm{~cm}^{-1}$ is generally associated with $\beta$-turns [41]; however, in the present case, the signature is weak. The amorphous fibroin spectrum has a broader, less defined line at the Amide III band, with more spread towards higher wavenumbers, in line with its disorder. Likewise, amorphous fibroin and, to a lesser extent, $B$. mori silk has a strong line at $1340 \mathrm{~cm}^{-1}$ due to $\delta_{s}\left(\mathrm{CH}_{3}\right)$ also associated with the Silk I form [43]. Signatures in the $1350-1420 \mathrm{~cm}^{-1}$ window likewise represent bending vibrations $\delta_{s}\left(\mathrm{CH}_{3}\right)$ for polypeptides in various conformations [45]. The spectral line at $1443 \mathrm{~cm}^{-1}$ corresponds to asymmetric bending vibrations of $\delta_{a s}\left(\mathrm{CH}_{3}\right)$ variety in $\beta$-sheets of both poly-alanine $(\mathrm{Ala})_{n}$ and Ala-Gly ${ }_{n}$ [41], with intensity being highest in $\beta$-sheet rich domestic white silk and lowest 
for amorphous fibroin. The similarly $\delta_{a s}\left(\mathrm{CH}_{3}\right)$ associated line at $1456 \mathrm{~cm}^{-1}$ is related to the more generic vibrations in alanine and valine.

The three distinct varieties of fibroin-based structures show distinct variations in the Amide I and Amide II regions that dominate the spectra. Each of these lines has a complex spectral composition due to conformational variations [43]. The major constituents of the Amide II band are assigned as $1508 \mathrm{~cm}^{-1}$ arising from $\beta$-sheets and $1546 \mathrm{~cm}^{-1}$ is associated with disordered fibroin, as is evidenced by its prevalence for the amorphous sample. Amide I band follows a similar distribution but shows an even more pronounced structure, with components at $\sim 1625 \mathrm{~cm}^{-1}$ representing $\beta$-sheets and $1648 \mathrm{~cm}^{-1}$ associated with irregular structures including random coil and extended chains [43]. In addition, other signatures reported in literature are associated with Silk I, type II $\beta$-turns (1647-1654 cm $\mathrm{cm}^{-1}$ ), $\alpha$-coils (1658-1664 $\mathrm{cm}^{-1}$ ) and turns and bends $1699 \mathrm{~cm}^{-1}$ [46] (unaffected by the $\beta$-sheet disrupting fibroin extraction procedure). The Amide I, II bands were found broader in transmission (not shown) for the fibers as compared with amorphous fibroin [22], which is consistent with a higher orientation arising due to drawing [47]. In the single beam transmittance, the signal is integrated across the diameter of the fiber, whereas the ATR signal penetrates only a few micrometers from the surface. It is well known that the silk fiber has a core and shell structure, and the shell is comprised of low molecular weight amides while the core is rich in extended (Ala-Gly) $n$ or (Ala) $n$ chains [47]. Differences between degummed crystalline fibers of domestic (white) and wild (brown) silk are the most distinguishable around the $1000 \pm 100 \mathrm{~cm}^{-1}$ region. It was likewise previously observed that strong polarisation dependence exists at this wavelength range suitable for identification of silk species [31].
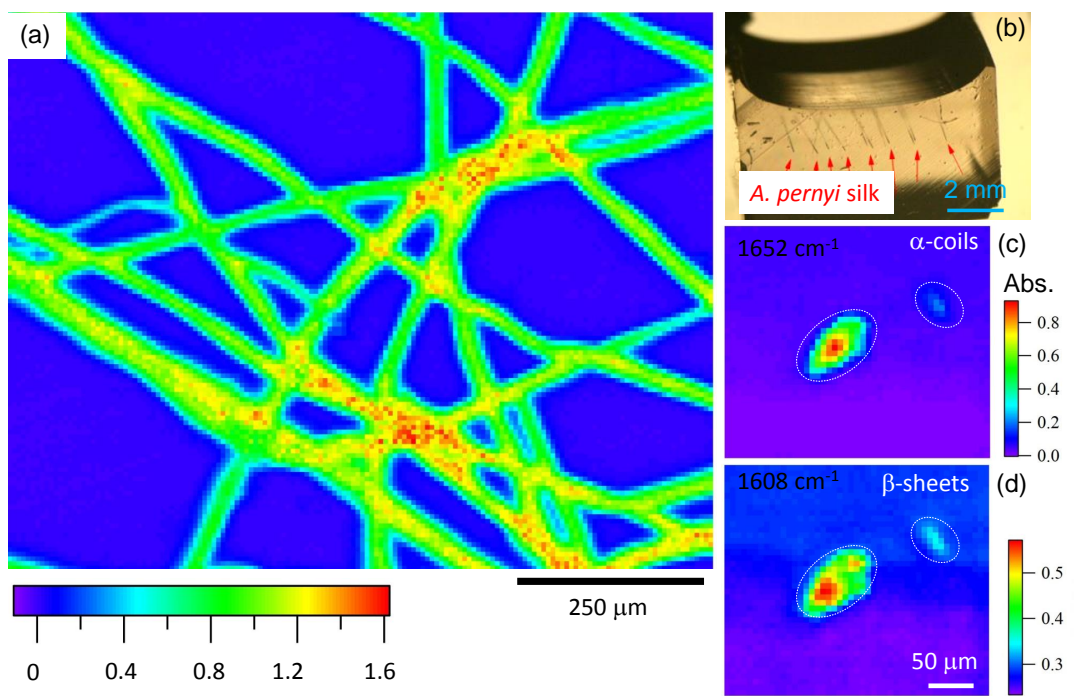

Figure 7. (a) natural A. pernyi silk absorbance IR image taken at $\sim 1660 \mathrm{~cm}^{-1}$ band (the region of $\alpha$-coils absorption); (b) photo of silk fibers embedded in a block of epoxy which was used for 5- $\mu$ m-wide microtome slices; (c,d) absorbance: axial cross sectional scans of $A$. pernyi fiber at $\beta$-sheet $\left(1608 \mathrm{~cm}^{-1}\right)$ and $\alpha$-coils $\left(1652 \mathrm{~cm}^{-1}\right)$ bands. Resolution was $6.25 \mu \mathrm{m}$. Oval contours show circumference of the fibers.

In order to shed further light on the internal structure of silk transmittance, IR imaging was performed for natural (not degummed) sericin rich brown silk fibers embedded in a thin $\mathrm{KBr}$ pallet. Spectroscopic maps were acquired at the $1660 \mathrm{~cm}^{-1}$ absorbance band preferentially caused by $\alpha$-coils, which were shown to be located towards the center of the fiber (Figure 7). The fiber is clearly distinguished from the $\mathrm{KBr}$ matrix. Furthermore, for the cross-sectional observation, the natural silk fibers were aligned and embedded into an epoxy adhesive (jER 828, Mitsubishi Chemical Co., Ltd., Kyoto, Japan) as shown in the Figure 7b. Fibers fixed in the epoxy matrix were cut in the 
perpendicular direction to the fiber by microtome (RV-240, Yamato Khoki Industrial Co., Ltd., Saitama, Japan). Figure 7c,d shows mid-IR transmittance spectral imaging of the $\sim 100 \mu \mathrm{m}$-diameter fiber and a smaller whisker (oval contours) taken at different wavenumbers. The difference in distribution at $1608 \mathrm{~cm}^{-1}$ and and $1652 \mathrm{~cm}^{-1}$ absorption bands of $\beta$-sheets and $\alpha$-coils, respectively, is clearly distinguishable. The $\alpha$ coils are located preferentially at the center of the fiber (as inferred from Figure 7), while $\beta$-sheets are distributed over the entire cross section and are present at the rim. This is consistent with observed differences between transmission and ATR measurements of silk absorbance showing slightly different absorption losses due to, respectively, throughout and evanescent propagation of light in those two modes of measurement [47].

\subsection{Vis-UV Range}

Fibroin based compounds have a low molar absorptivity in the visible to near-infrared spectral regions. Furthermore, the effects of water vapour are negligible at such wavelengths. Figure 8 shows the absorbance of domestic B. mori and wild $A$. pernyi silk fibers from the shortest UV wavelengths to near-IR spectral range, deduced from total hemispherical reflectance and transmittance spectra.

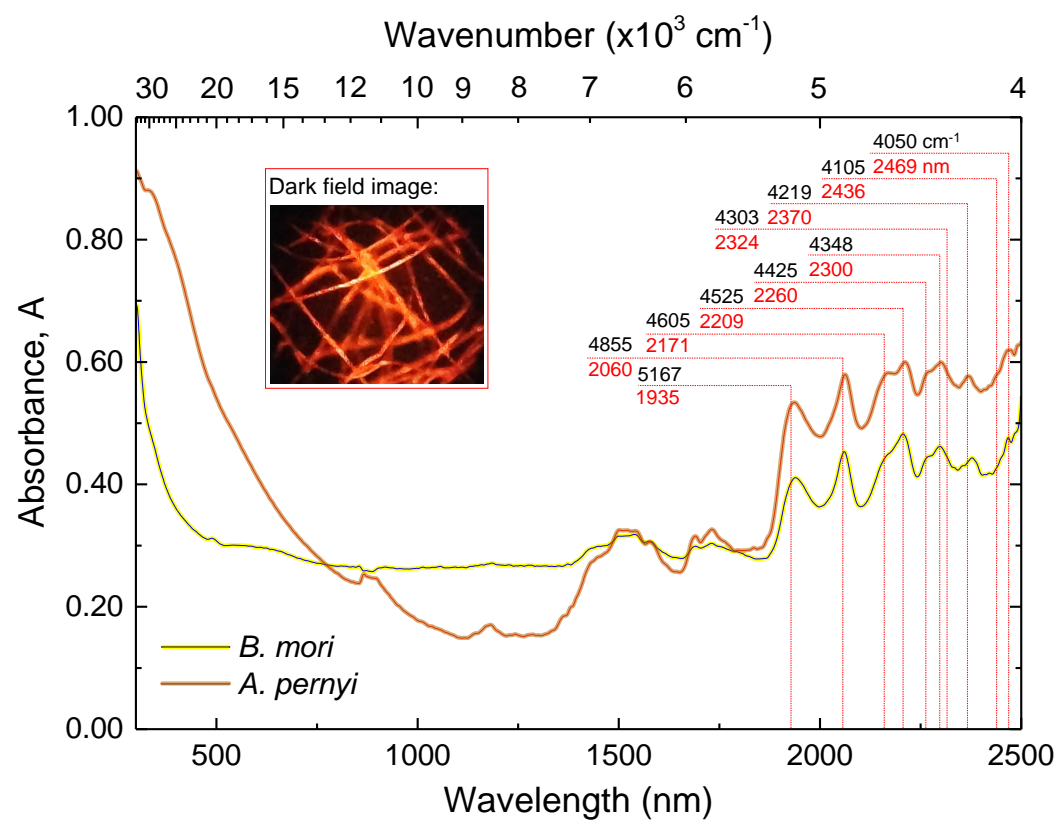

Figure 8. UV-near-IR Absorbance, $A$, spectra of degummed fibers of B. mori and A. pernyi. Inset shows an optical dark-field image of silk fibers. See text for the band assignment.

In the near-IR, absorbance lines in silk fibers can be attributed to the combination or overtone modes of various vibrations of the chemical bonds of the constituent peptides [48]. An example of such combination modes are the vibrations of aliphatic $\mathrm{C}-\mathrm{H}$ observed at $4010 \mathrm{~cm}^{-1} \mathrm{due}^{\text {to }}$ $v(\mathrm{C}-\mathrm{H})_{s} / \mathrm{C}-\mathrm{H}$ skeletal vibrations and at $4105 \mathrm{~cm}^{-1}$ due to combined $v(\mathrm{C}-\mathrm{H})_{a s} / \mathrm{C}-\mathrm{H}$ skeletal vibrations. Similarly, the lines at $4050 \mathrm{~cm}^{-1}$ and $4170 \mathrm{~cm}^{-1}$, respectively, are assigned to combination modes of $v(\mathrm{C}-\mathrm{H})_{s} / r(\mathrm{C}-\mathrm{H})$ and $v(\mathrm{C}-\mathrm{H})_{a s} / r(\mathrm{C}-\mathrm{H})$. The next group of four vibration combinations has similarly been tied to $v(\mathrm{C}-\mathrm{H}) / \delta(\mathrm{C}-\mathrm{H})$ motions in symmetric/symmetric $\left(4250 \mathrm{~cm}^{-1}\right)$, asymmetric/symmetric $\left(4303 \mathrm{~cm}^{-1}\right)$, symmetric/asymmetric $\left(4348 \mathrm{~cm}^{-1}\right)$ and asymmetric/asymmetric $\left(4425 \mathrm{~cm}^{-1}\right)$ configurations, respectively.

The spectral range between $4500-4900 \mathrm{~cm}^{-1}$ is especially informative, since it contains the combinations of various amide lines: Amide A and Amide III (4525 cm $\mathrm{cm}^{-1}$, Amide B and Amide II $\left(4605 \mathrm{~cm}^{-1}\right)$, Amide A and Amide II $\left(4855 \mathrm{~cm}^{-1}\right)$. Particularly, the Amide A and Amide III combination at $4525 \mathrm{~cm}^{-1}$ has been shown to be associated with $\beta$-sheets as it relates to the strength and prevalence 
of hydrogen bonds [49]. In contrast, Amide A and Amide II at $4855 \mathrm{~cm}^{-1}$ tied to random $\alpha$-coils [48,49]. Thereby, the larger fraction of $\beta$-sheets in B. mori over the wild silk variety observed in mid-IR spectra is corroborated by results in the near-IR.

The pronounced peak at $5167 \mathrm{~cm}^{-1}$ is reported as arising due to $v(\mathrm{O}-\mathrm{H})$ and $\delta(\mathrm{O}-\mathrm{H})$ combined vibrations [48]. Spectral features beyond $5200 \mathrm{~cm}^{-1}$ represent the first and second overtones of various hydrogen bonds. The spectra reveal low scattering losses at visible wavelengths, which would follow $\propto \lambda^{-4}$ Rayleigh scattering scaling. Regenerated silk fibroin can be used to make optical fibers with waveguiding losses $<0.1 \mathrm{~dB} / \mathrm{cm}$ (at $15,800 \mathrm{~cm}^{-1}$, or $633 \mathrm{~nm}$ in wavelength) comparable with polymethylmetacrilate (PMMA) and even original silk fibers perform as optical waveguides of $2.9 \mathrm{~dB} / \mathrm{mm}$ at visible wavelengths [8].

Significant absorption starts to dominate the spectra at UV wavelengths. Figure 9 shows a photoluminescence excitation spectrum (PLE) of degummed B. mori and A. pernyi fiber specimens. In both cases, a strong PL excitation band centered at the excitation wavelength of $15,800 \mathrm{~cm}^{-1}$ $(\sim 280 \mathrm{~nm})$ was present. It is typical for proteins in natural bio-materials and is related to $\pi \longrightarrow \pi^{*}$ transitions in amino acids that have aromatic rings such as tyrosine, phenylalanine, tryptophan and histidine. Residues of tyrosine in particular have been shown to account for a significant portion of absorption in the $15,800 \mathrm{~cm}^{-1}(280 \mathrm{~nm})$ region [50,51]. A remnant of sericin in the degummed fibers has a signature with its absorption band at 42,550-46,500 $\mathrm{cm}^{-1}$ (215-235 nm) (FWHM) wavelengths [52]. The bonds of most other peptides (including the main building blocks of fibroin-alanine, glycine and serine) absorb at wavelengths below $47,620 \mathrm{~cm}^{-1}(210 \mathrm{~nm})$; however, due to the abundance and variety of peptide bonds in biomaterials as well as overlapping absorption of other materials, this spectral range presents considerable analytical challenges [53].
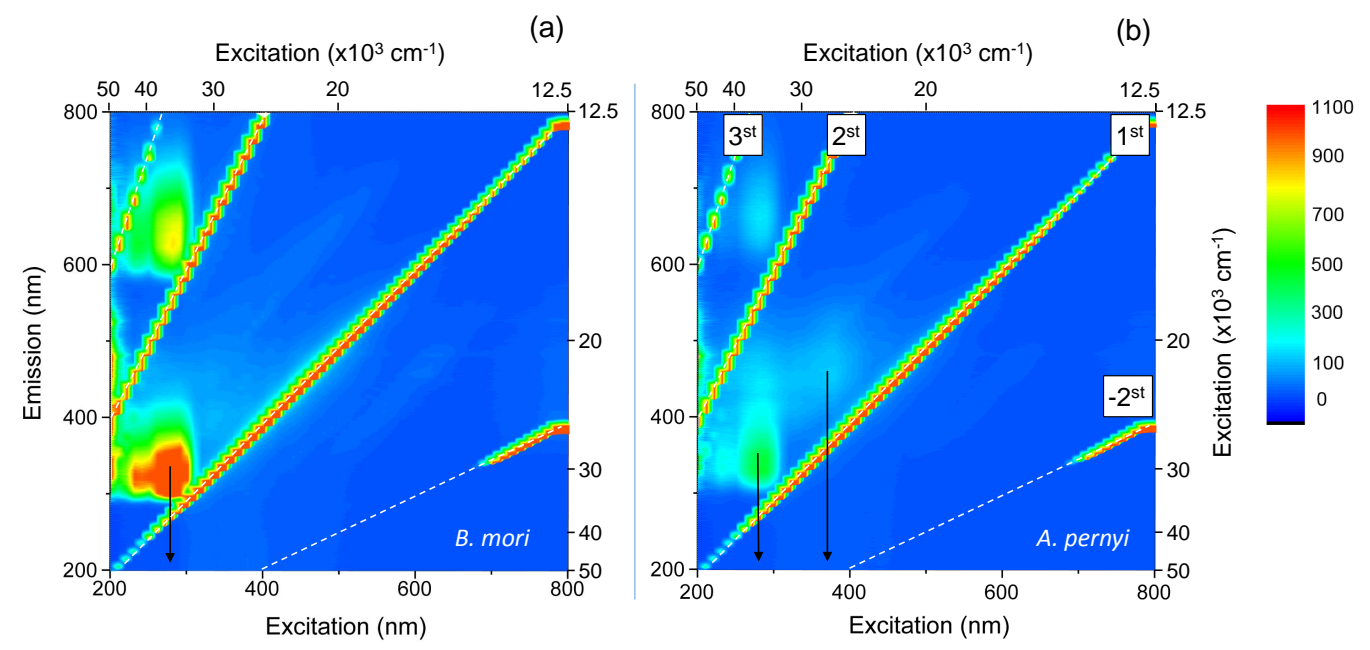

Figure 9. Photoluminescence excitation spectra (PLE) of degummed B. mori (a) and A. pernyi (b) silk fibers. The arrow in (a) shows center band excitation wavelength $\sim 35,714 \mathrm{~cm}^{-1}(\sim 280 \mathrm{~nm})$. The first and higher order diffraction artifacts are marked in (b). Photoluminescence (PL) at 15,625 $\mathrm{cm}^{-1}$ $(640 \mathrm{~nm})$ for the excitation of $\sim 35,714 \mathrm{~cm}^{-1}(\sim 280 \mathrm{~nm})$ is caused by second order diffraction.

In crystalline $A$. pernyi fibers, photoluminescence was relatively stronger than for the $B$. mori fibers and an additional PL band exists for the longer $\sim 26,315 \mathrm{~cm}^{-1}(\sim 380 \mathrm{~nm})$ excitation wavelengths. It has been reported that similar spectral signatures at $\sim 28,985 \mathrm{~cm}^{-1}(\sim 345 \mathrm{~nm})$ are observed for sericin solutions extracted from A. pernyi silks, with significant variations in UV spectral response of sericin from different silks [51]. The brown pigmentation of certain wild silks has also been hypothesised as resulting from $\sim 21,740 \mathrm{~cm}^{-1}(460 \mathrm{~nm})$ absorbing chromophores created through UV photo-oxidation of tyrosine residues, which may play a part in UV protection of the silk cocoon in its pupal stage [51]. 


\subsection{Discussion of the Band Assignment}

Absorption band assignments for the wild, domestic and spider silks in IR spectral range have been analysed using a multivariate approach [41] and were used in this study for IR absorption band assignment. It has been demonstrated by numerical modeling and spectral measurements that peptides form secondary structures and have spectrally broad absorption bands in the $100-500 \mathrm{~cm}^{-1}$ spectral region [54]. Typical bond energy in N-methylacetamide (the simplest peptide) is $120 \mathrm{~cm}^{-1}$, assigned to $\mathrm{CO} \cdots \mathrm{HN}$ intermolecular hydrogen bonding, and $201 \mathrm{~cm}^{-1}$ for the $\mathrm{C}-\mathrm{N}$ torsional vibration of the peptide bond [54]. However, the assignment to specific bonds is difficult due to substantive broadening occurring as a result of variations in the secondary structure.

Temperature of $77 \mathrm{~K}$ corresponds to the thermal energy of $53.5 \mathrm{~cm}^{-1}$. Since the absorption bands did not experience spectral narrowing (Figure 4), it was concluded that those vibrations are intrinsic to the structural components of the crystalline silk fibers and for amorphous fibroin.

It has been demonstrated that Raman active bands observed in scattering of $B$. mori silk at 1085,1232 , and $1667 \mathrm{~cm}^{-1}$ correspond to the absorption of random coils $\left(1085 \mathrm{~cm}^{-1}\right)$ and $\beta$-sheets, respectively. Hence, absorption and scattering have similarities in characteristic spectral bands. While bands at 412 and $260 \mathrm{~cm}^{-1}$ are ascribed to the $-\mathrm{SO}_{2}$ - moiety [55], the quartet of spectral signatures at around 250, 328, 427, and $553 \mathrm{~cm}^{-1}$, observed in $\beta$-sheet rich $B$. mori fibroin films, provide a closer correspondence [39]. The peak at $553 \mathrm{~cm}^{-1}$ is especially instructive as it is observed in (Ala-Gly) ${ }_{n}$ copolymers, but not in either poly-alanine or poly-glycine. The absence of an equivalent peak in A. pernyi fibers, where alanine and glycine rich regions are segregated, further reinforces this assignment. The low energy bands are also found at the silk-I polypeptide recognisably by signatures at about $1415,1105,950,930,865,260$, and $230 \mathrm{~cm}^{-1}$ [44]. The spectral features of $A$. pernyi fibers at $20 \mathrm{~cm}^{-1}$ and $67 \mathrm{~cm}^{-1}(0.6 \mathrm{THz}$ and $2 \mathrm{THz})$ are, most probably, related to the hydrogen bonding $\mathrm{O}-\mathrm{H} \cdots \mathrm{O}$ where interaction between the neighboring segments define the acoustic response of material in a similar way as in water, where a $70 \mathrm{~cm}^{-1}$ band has been ascribed to the third or fourth neighbor network response [56]. Since A. pernyi silk has a smaller amount of $\beta$-sheets [31] as compared with the B. mori silk which has $60 \%-65 \%$ crystallinity [57], the polymeric network is interacting via hydrogen bond linked segments. This defines spectroscopic differences at a low energy range of T-rays observed in experiments (Figure 1) and explains stretchability and contraction of silk [31]. In addition, significant differences between the spectral signatures of water insoluble fibers and soluble amorphous fibroin, uncovered in the $200-300 \mathrm{~cm}^{-1}$ far-IR spectral region, allow for the inference of important variations in secondary structure of this biopolymer.

\section{Conclusions}

Spectral absorbance of silk over $\sim 3.8$ decades in frequency $(3.8 / \lg 2=12.6$ octaves $)$ was measured ranging from THz $8 \mathrm{~cm}^{-1}(\lambda=1.25 \mathrm{~mm}, f=0.24 \mathrm{THz})$ to deep-UV $50 \times 10^{3} \mathrm{~cm}^{-1}(\lambda=200 \mathrm{~nm}$, $f=1500 \mathrm{THz}$ ). Whereas spectroscopy at the UV and visible ranges provides information about chemical composition, near- and mid-IR offers a wealth of information on the immediate environment of certain bonds, T-ray spectra are exquisitely sensitive to variations in secondary structure, albeit at the cost of specificity. Low energy T-ray bands at $\sim 243$ and $\sim 229 \mathrm{~cm}^{-1}$, probably related to CO-NH torsional vibrations [39] in domestic and wild degummed silk fibers, respectively, were observed and showed no spectral shift down to $78 \mathrm{~K}$ temperature. The difference between domestic and wild silk crystalline fibers was observed at $20 \mathrm{~cm}^{-1}$ and $67 \mathrm{~cm}^{-1}(0.6 \mathrm{THz}$ and $2 \mathrm{THz})$ bands, which correspond to the spectral region of acoustic response in hydrogen bonding networks.

High transmittance of silk up to $35,714 \mathrm{~cm}^{-1}(280 \mathrm{~nm})$ wavelengths and its birefringence makes it a promising material for micro-optical applications where polymerisable resists and resins usually have low transmittance. This can be found to be appealing for micro-fluidic and opto-genetic applications using aqueous and bio-tissue ambiance. Water solubility of silk, its biodegradability and in-body desorption are critically important for fabrication of bio-sensor platforms [58], implants, and wearable electronics which are fast developing using silk and can be better understood from 
spectroscopic analysis over a broad spectral range. Far-IR/THz spectroscopy bolsters the chemical fingerprinting capability afforded by shorter wavelength-based methods, as it is capable of probing the secondary structure of complex protein-based materials even in their natural states. In this study, silkworm spun fibers were investigated as a point for future reference. However, such broad spectrum analysis is useful in investigating the structural hierarchy of silk to guide bottom-up methods for self-assembly of fibroin-based artificial biocompatible functional materials.

Acknowledgments: This work was part of the Melbourne synchrotron beamtime proposal 10457, experiments carried out from 19 to 21 April 2016. Meguya Ryu is grateful for the travel grant from the Tokyo Institute of Technology. Junko Morikawa acknowledges the support of JSPS KAKENHI Grant No. 16K06768 and the support in part by "Materials research by Information Integration" Initiative (MI2I) project of the Support Program for the Starting Up Innovation Hub from the Japan Science and Technology Agency (JST). Saulius Juodkazis is grateful for partial support via the Australian Research Council DP130101205 Discovery project, Swinburne's startup grant for nanotechnology facility, and by the nanotechnology Ambassador fellowship program at the Melbourne Centre for Nanofabrication (MCN) in the Victorian Node of the Australian National Fabrication Facility (ANFF).

Author Contributions: Saulius Juodkazis and Junko Morikawa come up with the idea of experiments, Armandas Balčytis, Meguya Ryu, Xuewen Wang, Gediminas Seniutinas, Jingliang Li and Saulius Juodkazis carried out experiments at the Melbourne synchrotron on the beamline supervised by Dominique Appadoo, silk samples were prepared by Shan Du under supervision of Xungai Wang and Jingliang Li, T-ray time-domain spectroscopy characterisation was carried out by Fabio Novelli and Jeffrey Davis, measurements of FT-IR spectroscopy were accomplished by Meguya Ryu and Junko Morikawa, and UV-Vis-NIR spectroscopy measurements were performed by Armandas Balčytis. All the authors participated in discussion and analysis of the results and contributed to editing of the manuscript.

Conflicts of Interest: The authors declare no conflict of interest.

\section{References}

1. Mlnonl, G.; Zerbl, G. End Effects on Longitudinal Accordion Modes: Fatty Acids and Layered Systems. J. Phys. Chem. 1982, 86, 4791-4798.

2. Mizushima, S.; Shimanouchi, T. Raman frequencies of n-paraffin molecules. J. Am. Chem. Soc. 1949, 7, 1320-1324.

3. Doucet, J.; Denicolo, I.; Craievich, A. X-ray study of the rotator phase of the odd numbered paraffins $\mathrm{C}_{17} \mathrm{H}_{36}$, $\mathrm{C}_{19} \mathrm{H}_{40}$, and $\mathrm{C}_{21} \mathrm{H}_{44}$. J. Chem. Phys. 1981, 75, 1523.

4. Barnes, J.D.; Fanconi, B.M. Raman Spectroscopy, Rotational Isomerism, and the "Rotator" Phase Transition in n-Alkanes. J. Chem. Phys. 1972, 56, 5190-5192.

5. Chumakov, A.I.; Monaco, G.; Monaco, A.; Crichton, W.A.; Bosak, A.; Rüffer, R.; Meyer, A.; Kargl, F.; Comez, L.; Fioretto, D.; et al. Equivalence of the Boson Peak in Glasses to the Transverse Acoustic van Hove Singularity in Crystals. Phys. Rev. Lett. 2011, 106, 225501.

6. Png, G.M.; Falconer, R.J.; Abbott, D. Tracking Aggregation and Fibrillation of Globular Proteins Using Terahertz and Far-Infrared Spectroscopies. IEEE Trans. Terahertz Ssci. Technol. 2016, 6, 45.

7. Liu, X.; Zhang, K.Q. Silk Fiber-Molecular Formation Mechanism, Structure- Property Relationship and Advanced Applications. In Oligomerization of Chemical and Biological Compounds; Lesieur, C., Ed.; Intech: Rijeka, Croatia, 2014.

8. Kujala, S.; Mannila, A.; Karvonen, L.; Kieu, K.; Sun, Z. Natural Silk as a Photonics Component: A Study on Its Light Guiding and Nonlinear Optical Properties. Sci. Rep. 2016, 6, 22358.

9. Tao, H.; Kaplan, D.L.; Omenetto, F.G. Silk Materials: A Road to Sustainable High Technology. Adv. Mater. 2012, 24, 2824-2837.

10. Li, G.; Li, Y.; Cher, G.; He, J.; Han, Y.; Wang, X.; Kaplan, D.L. Silk-Based Biomaterials in Biomedical Textiles and Fiber-Based Implants. Adv. Healthc. Mater. 2015, 4, 1134-1151.

11. Kim, S.; Marelli, B.; Brenckle, M.A.; Mitropoulos, A.N.; Gil, E.S.; Tsioris, K.; Tao, H.; Kaplan, D.L.; Omenetto, F.G. All-water-based electron-beam lithography using silk as a resist. Nat. Nanotechnol. 2014, 9, 306-310

12. Balčytis, A.; Ryu, M.; Seniutinas, G.; Juodkazytè, J.; Cowie, B.C.C.; Stoddart, P.R.; Morikawa, J.; Juodkazis, S. Black-CuO: Surface-enhanced Raman scattering and infrared properties. Nanoscale 2015, 7, 18299-18304.

13. Sun, Y.L.; Li, Q.; Sun, S.M.; Huang, J.C.; Zheng, B.Y.; Chen, Q.D.; Shao, Z.Z.; Sun, H.B. Aqueous multiphoton lithography with multifunctional silk-centred bio-resists. Nat. Commun. 2015, 6, 8612. 
14. Kundu, B.; Rajkhowa, R.; Kundu, S.C.; Wang, X. Silk fibroin biomaterials for tissue regenerations. Adv. Drug Delivery Rev. 2013, 65, 457-470.

15. Hu, X.; Shmelev, K.; Sun, L.; Gil, E.S.; Park, S.H.; Cebe, P.; Kaplan, D.L. Regulation of Silk Material Structure by Temperature-Controlled Water Vapor Annealing. Biomacromolecules 2011, 12, 1686-1696.

16. Zhu, B.; Wang, H.; Leow, W.R.; Cai, Y.; Loh, X.J.; Han, M.Y.; Chen, X. Silk Fibroin for Flexible Electronic Devices. Adv. Mater. 2016, 28, 4250-4265.

17. Kalantar-Zadeh, K.; Oua, J.Z.; Daeneke, T.; Mitchell, A.; Sasaki, T.; Fuhrer, M.S. Two dimensional and layered transition metal oxides. Appl. Mater. Today 2016, 5, 73-89.

18. Fei, X.; Shao, Z.; Chen, X. Synthesis of hierarchical three-dimensional copper oxide nanostructures through a biomineralization-inspired approach. Nanoscale 2013, 5, 7991-7997.

19. Sheng, W.; Zhu, G.; Kaplan, D.L.; Cao, C.; Zhu, H.; Lu, Q. Silk-regulated hierarchical hollow magnetite/carbon nanocomposite spheroids for lithium-ion battery anodes. Nanotechnology 2015, 26, 115603.

20. Bai, S.; Zhang, X.; Lu, Q.; Sheng, W.; Liu, L.; Dong, B.; Kaplan, D.L.; Zhu, H. Reversible Hydrogel-Solution System of Silk with High Beta-Sheet Content. Nanotechnology 2014, 15, 3044-3051.

21. Cebe, P.; Hu, X.; Kaplan, D.L.; Zhuravlev, E.; Wurm, A.; Arbeiter, D.; Schick, C. Beating the Heat-Fast Scanning Melts Silk Beta Sheet Crystals. Sci. Rep. 2013, 3, 1130.

22. Morikawa, J.; Ryu, M.; Balčytis, A.; Seniutinas, G.; Fan, L.; Mizeikis, V.; Li, J.L.; Wang, X.W.; Zamengo, M.; Wang, X.; et al. Silk fibroin as water-soluble bio-resist and its thermal properties. RSC Adv. 2015, 6, 11863-11869.

23. Hebling, J.; Yeh, K.L.; Hoffmann, M.C.; Bartal, B.; Nelson, K.A. Generation of high-power terahertz pulses by tilted-pulse-front excitation and their application possibilities. J. Opt. Soc. Am. B 2008, 25, B6-B19.

24. Novelli, F.; Chon, J.W.M.; Davis, J.A. Terahertz thermometry of gold nanospheres in water. Opt. Lett. 2016, 41, 5801-5804.

25. Blanchard, F.; Sharma, G.; Razzari, L.; Ropagnol, X.; Bandulet, H.C.; Vidal, F.; Morandotti, R.; Kieffer, J.C.; Ozaki, T.; Tiedje, H.; et al. Generation of Intense Terahertz Radiation via Optical Methods. IEEE J. Sel. Top. Quant. Electron. 2011, 17, 5-16.

26. Juodkazis, S.; Mizeikis, V.; Matsuo, S.; Ueno, K.; Misawa, H. Three-Dimensional Micro- and Nano-Structuring of Materials by Tightly Focused Laser Radiation. Bull. Chem. Soc. Jpn. 2008, 81, 411-448.

27. Keten, S.; Xu, Z.; Ihle, B.; Buehler, M.J. Nanoconfinement controls stiffness, strength and mechanical toughness of $\beta$-sheet crystals in silk. Nat. Mater. 2010, 10, 359-367.

28. Sezutsu, H.; Yukuhiro, K. Dynamic Rearrangement Within the Antheraea pernyi Silk Fibroin Gene Is Associated with Four Types of Repetitive Units. J. Mol. Evol. 2000, 51, 329-338.

29. Zhang, Y.; Yang, H.; Shao, H.; Hu, X. Antheraea pernyi Silk Fiber: A Potential Resource for Artificially Biospinning Spider Dragline Silk. J. Biomed. Biotechnol. 2010, 2010, 683962.

30. Zuo, B..; Leigen, L.; Zhengyu, W. Effect on Properties of Regenerated Silk Fibroin Fiber Coagulated with Aqueous Methanol/Ethanol. J. Appl. Polym. Sci. 2007, 106, 53-59.

31. Ling, S.; Qi, Z.; Knight, D.P.; Huang, Y.; Huang, L.; Zhou, H.; Shao, Z.; Chen, X. Insight into the structure of single Antheraea pernyi silkworm fibers using synchrotron FTIR microspectroscopy. Biomacromolecules 2013, 14, 1885-1892.

32. Falconer, R.J.; Markelz, A.G. Terahertz spectroscopic analysis of peptides and proteins. J. Infrared Milli. Terahz. Waves 2012, 33, 973-988.

33. Cho, S.Y.; Yun, Y.S.; Lee, S.; Jang, D.; Park, K.Y.; Kim, J.K.; Kim, B.H.; Kang, K.; Kaplan, D.L.; Jin, H.J. Carbonization of a stable $\beta$-sheet-rich silk protein into a pseudographitic pyroprotein. Nat. Commun. 2015, 6, 7145 .

34. Zelsmann, H.R. Temperature dependence of the optical constants for liquid $\mathrm{H}_{2} \mathrm{O}$ and $\mathrm{D}_{2} \mathrm{O}$ in the far IR region. J. Mol. Struct. 1995, 350, 95-114.

35. Brubach, J.B.; Mermet, A.; Filabozzi, A.; Gerschel, A.; Roy, P. Signatures of the hydrogen bonding in the infrared bands of water. J. Chem. Phys. 2005, 122, 184509.

36. Yazawa, K.; Ishida, K.; Masunaga, H.; Hikima, T.; Numata, K. Influence of Water Content on the $\beta$-Sheet Formation, Thermal Stability, Water Removal, and Mechanical Properties of Silk Materials. Biomacromolecules 2016, 17, 1057-1066.

37. Medcraft, C.; McNaughton, D.; Thompson, C.D.; Appadoo, D.; Bauerecker, S.; Robertson, E.G. Size and temperature dependence in the far-IR spectra of water ice particles. Astrophys. J. 2012, 758, 17. 
38. Sun, C.Q.; Zhang, X.; Fu, X.; Zheng, W.; Kuo, J.L.; Zhou, Y.; Shen, Z.; Zhou, J. Density and Phonon-Stiffness Anomalies of Water and Ice in the Full Temperature Range. J. Phys. Chem. Lett. 2013, 4, 3238-3244.

39. Itoh, K.; Nakahara, T.; Shimanouchi, T.; Oya, M.; Uno, K.; Iwakura, Y. Far-infrared spectra of polyalanines with $\alpha$-helical and $\beta$-form structures. Biopolymers 1968, 6, 1759-1766.

40. Falconer, R.J.; Zakaria, H.A.; Fan, Y.Y.; Bradley, A.P.; Middelberg, A.P. Far-Infrared Spectroscopy of Protein Higher-Order Structures. Appl. Spectrosc. 2010, 64, 1259-1264.

41. Boulet-Audet, M.; Vollrath, F.; Holland, C. Identification and classification of silks using infrared spectroscopy. J. Exp. Biol. 2015, 218, 3138-3149.

42. Papadopoulos, P.; Sölter, J.; Kremer, F. Structure-property relationships in major ampullate spider silk as deduced from polarized FTIR spectroscopy. Eur. Phys. J. E 2007, 24, 193-199.

43. Taddei, P.; Monti, P. Vibrational infrared conformational studies of model peptides representing the semicrystalline domains of Bombyx mori silk fibroin. Biopolymers 2005, 78, 249-258.

44. Monti, P.; Taddei, P.; Freddi, G.; Ohgo, K.; Asakura, T. Vibrational 13C-Cross-Polarization/Magic Angle Spinning NMR Spectroscopic and Thermal Characterization of Poly(alanine-glycine) as Model for Silk I Bombyx mori Fibroin. Biopolymers 2003, 72, 329-338.

45. Moore, W.H.; Krimm, S. Vibrational analysis of peptides, polypeptides, and proteins. II. $\beta$-poly(L-alanine) and $\beta$-poly(L-alanylglycine). Biopolymers 1976, 15, 2465-2483.

46. Lu, Q.; Hu, X.; Wang, X.; Kluge, J.A.; Lu, S.; Cebe, P.; Kaplan, D.L. Water-insoluble silk films with silk I structure. Acta Biomater. 2010, 6, 1380-1387.

47. Kazarian, S.G.; Chan, K.L.A. ATR-FTIR spectroscopic imaging: recent advances and applications to biological systems. Analyst 2013, 138, 1940-1951.

48. Mo, C.; Wu, P.; Chen, X.; Shao, Z. Near-Infrared Characterization on the Secondary Structure of Regenerated Bombyx Mori Silk Fibroin. Appl. Spectrosc. 2006, 60, 1438-1441.

49. Yuan, Q.; Yao, J.; Huang, L.; Chen, X.; Shao, Z. Correlation between structural and dynamic mechanical transitions of regenerated silk fibroin. Polymer 2010, 51, 6278-6283.

50. Millington, K.R. Diffuse reflectance spectroscopy of fibrous proteins. Amino Acids 2012, 43, 1277-1285.

51. Kaur, J.; Rajkhowa, R.; Tsuzuki, T.; Millington, K.; Zhang, J.; Wang, X. Photoprotection by Silk Cocoons. Biomacromolecules 2013, 14, 3660-3667.

52. Pushpa, A.; Goutham Vishnu, B.; Thippa Reddy, K.S. Preparation of nano silk sericin based hydrogels from silk industry waste. J. Environ. Res. Develop. 2013, 8, 243-253.

53. Noble, J.E.; Bailey, M.J.A. Chapter 8 Quantitation of Protein. In Guide to Protein Purification, 2nd ed.; Burgess, R.R., Deutscher, M.P., Eds.; Methods in Enzymology; Academic Press: Cambridge, MA, USA, 2009; Volume 463, pp. 73-95.

54. Ding, T.; Huber, T.; Middelberg, A.P.; Falconer, R.J. Characterization of Low-Frequency Modes in Aqueous Peptides Using Far-Infrared Spectroscopy and Molecular Dynamics Simulation. J. Phys. Chem. A 2011, 115, 11559-11565.

55. Aguayo, T.; Garrido, C.; Clavijo, R.E.; Gómez-Jeria, J.S.; Monasterio, C.A.; Icaza, M.; Moraga, F.E.; Vallette, M.M.C. Raman and surface enhanced Raman scattering of a black dyed silk. J. Raman Spectrosc. 2013, 44, 1238-1245.

56. Walrafen, G.E. Raman Spectrum of Water: Transverse and Longitudinal Acoustic Modes below $300 \mathrm{~cm}^{-1}$ and Optic Modes above $300 \mathrm{~cm}^{-1}$. J. Phys. Chem. 1990, 94, 2237-2239.

57. Hu, X.; Kaplan, D.; Cebe, P. Determining Beta-Sheet Crystallinity in Fibrous Proteins by Thermal Analysis and Infrared Spectroscopy. Macromolecules 2006, 39, 6161-6170.

58. Maximova, K.; Wang, X.W.; Balčytis, A.; Fan, L.; Li, J.; Juodkazis, S. Silk patterns made by direct femtosecond laser writing. Biomicrofluidics 2016, 10, 054101.

(C) 2017 by the authors. Licensee MDPI, Basel, Switzerland. This article is an open access article distributed under the terms and conditions of the Creative Commons Attribution (CC BY) license (http://creativecommons.org/licenses/by/4.0/). 